\title{
Superconductivity in Quasi-One-Dimensional $\mathrm{K}_{2} \mathrm{Cr}_{3} \mathrm{As}_{3}$ with Significant Electron Correlations
}

\author{
Jin-Ke Bao, ${ }^{1}$ Ji-Yong Liu, ${ }^{2}$ Cong-Wei Ma, ${ }^{1}$ Zhi-Hao Meng, ${ }^{1}$ Zhang-Tu Tang, ${ }^{1}$ Yun-Lei Sun, ${ }_{1}^{1}$ Hui-Fei Zhai, ${ }^{1}$ \\ Hao Jiang, ${ }^{1}$ Hua Bai, ${ }^{1}$ Chun-Mu Feng, ${ }^{1}$ Zhu-An Xu, ${ }^{1,3,4}$ and Guang-Han Cao ${ }^{1,3,4, *}$ \\ ${ }^{1}$ Department of Physics, Zhejiang University, Hangzhou 310027, China \\ ${ }^{2}$ Department of Chemistry, Zhejiang University, Hangzhou 310027, China \\ ${ }^{3}$ State Key Lab of Silicon Materials, Zhejiang University, Hangzhou 310027, China \\ ${ }^{4}$ Collaborative Innovation Centre of Advanced Microstructures, Nanjing 210093, China
}

(Received 13 January 2015; published 9 February 2015)

\begin{abstract}
We report the discovery of bulk superconductivity (SC) at $6.1 \mathrm{~K}$ in a quasi-one-dimensional chromium pnictide $\mathrm{K}_{2} \mathrm{Cr}_{3} \mathrm{As}_{3}$ that contains $\left[\left(\mathrm{Cr}_{3} \mathrm{As}_{3}\right)^{2-}\right]_{\infty}$ double-walled subnanotubes with face-sharing $\mathrm{Cr}_{6 / 2}$ $\left(\mathrm{As}_{6 / 2}\right)$ octahedron linear chains in the inner (outer) wall. The material has a large electronic specific-heat coefficient of $70-75 \mathrm{~mJ} \mathrm{~K}^{-2} \mathrm{~mol}^{-1}$, indicating significantly strong electron correlations. A linear temperature dependence of resistivity in a broad temperature range from 7 to $300 \mathrm{~K}$ is observed, which suggests non-Fermi liquid behavior of the material. Unconventional SC is preliminarily manifested by the estimated upper critical field exceeding the Pauli limit by a factor of 3-4. The title compound represents a rare example that possibly unconventional SC emerges in a quasi-1D system with strong electron correlations.
\end{abstract}

DOI: 10.1103/PhysRevX.5.011013

\section{INTRODUCTION}

Electron correlations and reduced dimensionality, as central issues in contemporary condensed matter physics, play an important role in producing novel superconductivity (SC) in crystalline materials. This is prominently exemplified in quasi-two-dimensional cuprates [1], strontium ruthenate [2], and ferroarsenides [3], in which the correlated $d$ electrons are believed to be essential for the appearance of unconventional SC. As the dimensionality is further reduced to the quasi-1D scenario, however, SC occurs infrequently, mainly because of Peierls instability $[4,5]$. Representative examples of quasi-1D superconductors include earlier organic Bechgaard salts [6] and purple molybdenum bronze $\mathrm{Li}_{0.9} \mathrm{Mo}_{6} \mathrm{O}_{17}$ [7], where the $\mathrm{C} 2 p$ electrons and the Mo $4 d$ electrons are responsible for SC. Since $3 d$-transition elements generally bear stronger electron correlations, it is of great interest if a $3 d$-element-based quasi-1D compound would superconduct as well. The $\mathrm{Cu}$-based ladder material, as a crossover from 1D to 2D, was theoretically predicted [8] and experimentally confirmed [9] to superconduct, although under high pressures. Unfortunately, the SC actually locates in the quasi-2D regime due to a dimension crossover [10].

\footnotetext{
"ghcao@zju.edu.cn
}

Published by the American Physical Society under the terms of the Creative Commons Attribution 3.0 License. Further distribution of this work must maintain attribution to the author(s) and the published article's title, journal citation, and DOI.
Subject Areas: Condensed Matter Physics, Strongly Correlated Materials, Superconductivity

It was revealed that both cuprate and iron-based hightemperature superconductors may bear 1D features, such as stripes [11] and nematicity [12], albeit of their quasi-2D crystal structures. Thus, investigations on SC in quasi-1Dcorrelated electron systems may shed light on the longstanding puzzle of the unconventional superconducting mechanism because of the inherent simplicity of one dimensionality. Additional interest for pursuing a quasi1D material with interacting electrons comes from the possible realization of Luttinger liquid, in which an exotic spin-charge separation is expected [13].

Here, we report synthesis and characterizations of a chromium arsenide, $\mathrm{K}_{2} \mathrm{Cr}_{3} \mathrm{As}_{3}$, which explicitly shows a quasi-1D crystal structure featured by well-separated $\left[\left(\mathrm{Cr}_{3} \mathrm{As}_{3}\right)^{2-}\right]_{\infty}$ chains in the crystalline lattice. Strong electron correlations are indicated by the large electronic specific-heat coefficient. Remarkably, the new material hosts novel normal-state and superconducting properties that point to unconventional SC.

\section{EXPERIMENTAL METHODS}

\section{A. Sample synthesis}

Single crystals of $\mathrm{K}_{2} \mathrm{Cr}_{3} \mathrm{As}_{3}$ are grown by spontaneous nucleation in a high-temperature solution with a self-flux of KAs under vacuum. First, KAs (CrAs) is prepared by the reaction of the stoichiometric mixture of potassium pieces (99.95\%), chromium powder (99.95\%), and arsenic powder $(99.999 \%)$ at $473(973) \mathrm{K}$ for $16 \mathrm{~h}$ in evacuated quartz tubes. Caution should be taken to avoid the possible violent chemical reactions, and it is highly recommended that the 
(a)

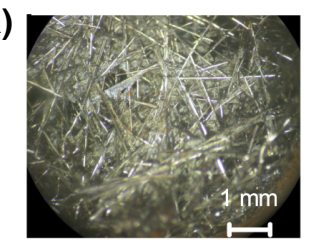

(b)

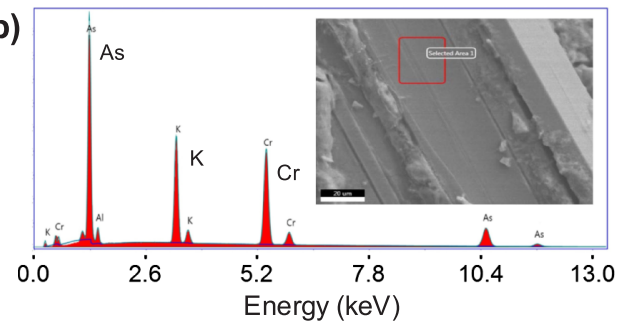

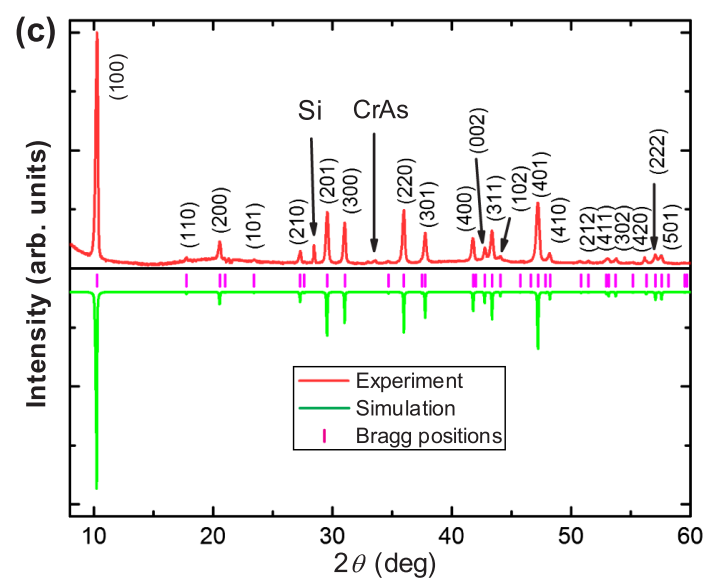

FIG. 1. Characterizations of the $\mathrm{K}_{2} \mathrm{Cr}_{3} \mathrm{As}_{3}$ samples. (a) Morphology of a batch of the as-grown crystals under an optical microscope (left: top view; right: side view). (b) A typical energy-dispersive X-ray spectrum with electron beams focused on the selected area (marked in the inset) of the as-grown crystals. A small amount of the element Al comes from the sample holder. (c) Powder x-ray diffraction of the polycrystalline samples indexed by the hexagonal unit cell determined from the single-crystal diffractions. Si is added as an internal standard. Only a small amount of the CrAs impurity is identified. The simulated pattern (with the consideration of preferential orientations of crystalline grains) well reproduces the experimental data.

initial heating should be very slow. Second, the presynthesized CrAs and KAs powders are mixed in a molar ratio of $1: 6$, namely, $\mathrm{K}: \mathrm{Cr}: \mathrm{As}=6: 1: 7$, and the mixtures are loaded in an alumina crucible. The alumina crucible (covered with a cap) is jacketed in a Ta tube, and the welded Ta tube is finally sealed in an evacuated quartz ampoule. The sample-loaded quartz ampoule is heated up to $1273 \mathrm{~K}$ holding for $24 \mathrm{~h}$ in a furnace, followed by cooling down to $923 \mathrm{~K}$ at a rate of $2 \mathrm{~K} / \mathrm{h}$. Shiny needlelike crystals with a typical size of $2 \times 0.1 \times 0.05 \mathrm{~mm}$ are harvested, as shown in Fig. 1(a). The polycrystalline sample is synthesized by a solid-state reaction in vacuum using the three elements as the starting materials. The stoichiometric ratio of $\mathrm{K}_{2} \mathrm{Cr}_{3} \mathrm{As}_{3}$ is taken. After the initial reaction, the mixtures are ground, and then pressed into a pellet. The pellet is finally sintered in vacuum at 973$1173 \mathrm{~K}$, holding for $24 \mathrm{~h}$. The resulted sample is dense, and it is suitable for the electrical resistivity and specific-heat measurements. Note that most of the procedures are carried out in an argon-filled glovebox with the water and oxygen content below $0.1 \mathrm{ppm}$. The synthesized samples are very reactive in air and easily deteriorate at ambient conditions. Thus, it is important to avoid exposure to air as much as possible while handling samples.

\section{B. Composition determination}

The chemical composition of the as-grown single crystals is measured by energy-dispersive $\mathrm{x}$-ray spectroscopy (EDS) with an AMETEK (C) EDAX (Model Octane Plus) spectrometer, equipped in a field-emission scanning electron microscope (Hitachi S-4800). The typical EDS spectrum is shown in Fig. 1(b). Through multiple measurements with a control experiment [see details in the Supplemental
Material (SM) [14]], the chemical composition of the asgrown crystals is determined to be $\mathrm{K}_{1.82(19)} \mathrm{Cr}_{3.00} \mathrm{As}_{2.99(12)}$, which is consistent with the ideal formula of $\mathrm{K}_{2} \mathrm{Cr}_{3} \mathrm{As}_{3}$ derived from the crystal structure.

\section{Structural determination}

The crystal structure is determined by single-crystal x-ray diffraction on an Xcalibur, Atlas, Gemini ultradiffractometer. Details of the experiment and analysis are presented in the SM [14]. The result indicates that the most probable structure at room temperature and below has a space group of $P \overline{6} m 2$ (No. 187). The obtained crystallographic structural data as well as the related bond lengths and bond angles are tabulated in Tables S3-S5 in the SM [14].

Powder $\mathrm{x}$-ray diffraction is carried out at room temperature on a PANalytical x-ray diffractometer (Empyrean Series 2) with a monochromatic $\mathrm{CuK}_{\alpha 1}$ radiation. To avoid a reaction with air, a thin layer of Apiezon $N$ grease is used to carefully coat the sample's surface. A small amount of $\mathrm{Si}$ powder is added as the internal standard reference material. As shown in Fig. 1(c), the powder x-ray diffraction peaks, both in positions and intensities, are quantitatively consistent with the simulated profile using the crystal structure data in Tables S3 and S4 of the SM [14]. This clearly indicates the same phase for the single crystals and polycrystals.

\section{Physical property measurements}

We employ a magnetic property measurement system (MPMS-5, Quantum Design) to measure the magnetic property. Both single crystals (a bundle of the crystal sticks) and bulk polycrystals are measured under an applied field of $H=10$ Oe with zero-field-cooling and 
field-cooling protocols. The electrical resistivity and the specific-heat capacity are measured for the polycrystalline samples, using a standard four-terminal method and a relaxation technique, respectively, on a physical properties measurement system (PPMS-9, Quantum Design). The heat capacity from the sample holder and grease is deducted. Note that our preliminary attempt to measure on a stick of single crystal is unsuccessful and/or unsatisfactory because of its tiny mass (for the magnetic and specific-heat measurements) or its high reactivity (for the resistivity measurement).

\section{RESULTS AND DISCUSSIONS}

\section{A. Crystal structure}

$\mathrm{K}_{2} \mathrm{Cr}_{3} \mathrm{As}_{3}$ crystallizes in a hexagonal lattice with $a=9.9832(9) \AA, c=4.2304(4) \AA$, and the most probable space group of $P \overline{6} m 2$ (No. 187) at room temperature [14]. The constituent atoms locate in the two crystalline planes with $z=0$ and $z=0.5$, as illustrated in Fig. 2(a). The prominent structural unit is the 1D negatively charged "chains," shown in Fig. 2(b). The $\left[\left(\mathrm{Cr}_{3} \mathrm{As}_{3}\right)^{2-}\right]_{\infty}$ chains are actually double-walled subnanotubes (DWSTs) of $0.58 \mathrm{~nm}$ outer diameter, in which face-sharing $\mathrm{Cr}_{6}\left(\mathrm{As}_{6}\right)$ octahedron tubes constitute the inner (outer) wall. $\mathrm{The}^{\mathrm{Cr}_{6 / 2}}$ "monomer" is basically a regular octahedron with $\mathrm{Cr}-\mathrm{Cr}$ bond lengths varying from 2.61 to 2.69(1) $\AA$. These $\mathrm{Cr}-\mathrm{Cr}$ bond distances are close to that $(2.50 \AA)$ in $\mathrm{Cr}$ metal, but much larger than twice $\left(1.46 \AA\right.$ ) the radius of $\mathrm{Cr}^{2+}$, indicating virtually metallic bonding among $\mathrm{Cr}$ atoms. Apart from the metallic bonding with six neighboring $\mathrm{Cr}$ atoms, each $\mathrm{Cr}$ atom also behaves like a cation (the apparent valence of $\mathrm{Cr}$ is +2.33 if assuming $\mathrm{K}^{+}$and $\mathrm{As}^{3-}$ ), which ionically bonds with four arsenic anions [see top illustration in Fig. 2(c)].
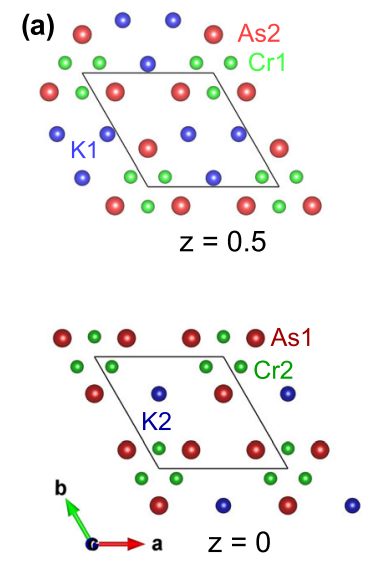

(b)
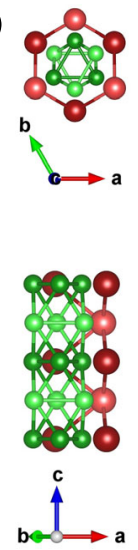

(c)
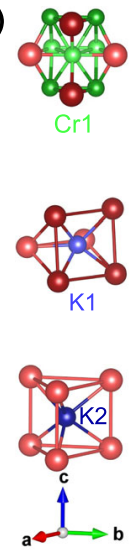

FIG. 2. Crystal structure of $\mathrm{K}_{2} \mathrm{Cr}_{3} \mathrm{As}_{3}$. (a) The constituted atoms locate in the two crystalline planes with $z=0$ and $z=0.5$. (b) The structure of $\left[\left(\mathrm{Cr}_{3} \mathrm{As}_{3}\right)^{2-}\right]_{\infty}$ double-walled subnanotubes (top: top view; bottom: side view with removal of the outer wall in the left front). (c) Chemical bonding of Cr1 (top), K1 (middle), and $\mathrm{K} 2$ (bottom).
The DWSTs are well separated by columns of the K counterions; hence, the inter-DWST coupling is expected to be much weaker than the intra-DWST interactions. There are two distinct crystallographic sites for potassium. K1 is coordinated with $\mathrm{As}_{6}$ triangular prisms horizontally [the middle of Fig. 2(c)]. K2 is also bonded with $\mathrm{As}_{6}$ triangular prisms, but vertically [see bottom illustration of Fig. 2(c)], and there are six additional arsenic anions surrounded farther within the same $a b$ plane (not shown).

After solving the crystal structure, we became aware of a similar structure in the $\mathrm{AkMo}_{3} \mathrm{Ch}_{3}$ or $\mathrm{Ak}_{2} \mathrm{Mo}_{6} \mathrm{Ch}_{6}$ ( $\mathrm{Ak}=$ alkali metals, $\mathrm{Tl}$, and $\mathrm{In} ; \mathrm{Ch}=$ chalcogen elements) series [15,16]. Although the 1D structural unit $\left[\left(\mathrm{Mo}_{3} \mathrm{Ch}_{3}\right)^{-}\right]_{\infty}$ is isostructural to the DWST of $\left[\left(\mathrm{Cr}_{3} \mathrm{As}_{3}\right)^{2-}\right]_{\infty}$, the former rotates a small angle along the $c$ axis, relative to the orientation of the latter. This leads to a different space group, $\mathrm{P}_{3} \mathrm{~m}$ (No. 176), for $\mathrm{AkMo}_{3} \mathrm{Ch}_{3}$. The $A k$ atoms occupy only the $(1 / 3,2 / 3,0)$ and $(2 / 3,1 / 3,1 / 2)$ sites, which gives $1: 3: 3$ stoichiometry. Another important difference lies in the slight distortion of the $\left[\left(\mathrm{Cr}_{3} \mathrm{As}_{3}\right)^{2-}\right]_{\infty}$ DWSTs, accompanied with the absence of the inversion center in $\mathrm{K}_{2} \mathrm{Cr}_{3} \mathrm{As}_{3}$.

\section{B. Superconductivity}

Figure 3(a) shows the temperature dependence of magnetic susceptibility $\chi(T)$ for a bundle of the crystal sticks (the sticks are mostly parallel to the external field) and the bulk polycrystalline sample of $\mathrm{K}_{2} \mathrm{Cr}_{3} \mathrm{As}_{3}$. A sharp diamagnetic transition is seen at $T_{c}=6.1 \mathrm{~K}$. The volume fractions (scaled by $4 \pi \chi$ ) of magnetic shielding, measured in the zero-field-cooling mode, exceed $100 \%$ at $2 \mathrm{~K}$ (they are approximately $100 \%$ after a correction of the demagnetization effect), suggesting bulk SC. On the other hand, the volume fractions of magnetic repulsion, reflected by the field-cooling data, are much smaller mainly because of the magnetic flux pinning in the process of cooling down under magnetic fields. The sharpness of the superconducting transitions, which is also shown in the following measurements, suggests sufficiently strong interchain couplings, albeit of the obvious quasi-1D structure.

Figure 3(b) shows the temperature dependence of electrical resistivity $\rho(T)$ measured with the polycrystalline sample [17]. Remarkably, the resistivity follows with $\rho(T)=\rho_{0}+A T \quad$ with $\rho_{0}=0.195 \mathrm{~m} \Omega \mathrm{cm} \quad$ and $A=$ $0.0067 \mathrm{~m} \Omega \mathrm{cm} \mathrm{K}^{-1}$ from 7 to $300 \mathrm{~K}$ [18]. This is reminiscent of the $T$-linear resistivity in optimally doped cuprate superconductors [19], iron pnictide superconductors [20], and $f$-electron systems [21], which is regarded as one of the hallmarks of non-Fermi-liquid behavior [22]. Owing to the quasi-1D characteristic here, the origin of the $T$-linear resistivity, if proven to be intrinsic, could be related to the possible Luttinger liquid [13,23]. SC is confirmed by the resistivity drop below $6.1 \mathrm{~K}$, as clearly seen in the top left-hand inset of Fig. 3(b). 

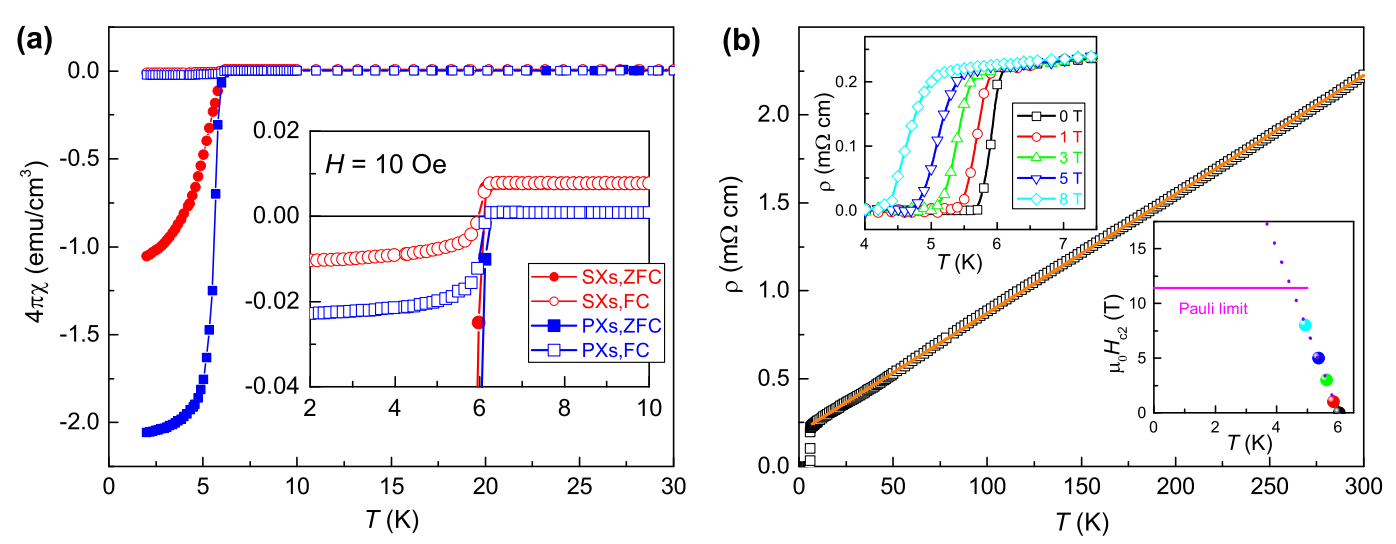

FIG. 3. Superconductivity in $\mathrm{K}_{2} \mathrm{Cr}_{3} \mathrm{As}_{3}$. (a) Temperature dependence of dc magnetic susceptibility for $\mathrm{K}_{2} \mathrm{Cr}_{3} \mathrm{As}_{3}$ single crystals (SXs) and polycrystals (PXs). The demagnetization effect was not taken into consideration. ZFC and FC denote zero-field cooling and field cooling, respectively. The inset magnifies the FC curves. (b) Temperature dependence of resistivity for the $\mathrm{K}_{2} \mathrm{Cr}_{3} \mathrm{As}_{3} \mathrm{polycrystalline}$ sample. The orange line is the linear fit for the normal state. The upper left-hand inset shows the superconducting transitions under different magnetic fields up to $8 \mathrm{~T}$, from which the upper critical field $\left(H_{c 2}\right)$ is derived (shown in the lower right-hand inset). The Pauli limit for $H_{c 2}$ is marked by the horizontal line.

Upon applying the magnetic field, the superconducting transition moves slowly to lower temperatures. The upper critical field $\left(H_{c 2}\right)$ is then determined using the common criteria of $90 \%$ of the extrapolated normal-state resistivity at the superconducting transition. The lower right-hand inset in Fig. 3(b) plots the obtained $H_{c 2}$ as a function of temperature, showing that $H_{c 2}$ increases very steeply with decreasing temperature. The absolute value of the initial slope, $-\mu_{0}\left(d H_{c 2} / d T\right)_{T \rightarrow T_{c}}$, is as high as $7.43 \mathrm{~T} / \mathrm{K}$. By a simple linear extrapolation, the zero-temperature upper critical field, $\mu_{0} H_{c 2}(0)$, is estimated to be $44.7 \mathrm{~T}$. If applying the Werthammer-Helfand-Hohenberg model [24], in which only the orbital effect is taken into account, the $\mu_{0} H_{c 2}(0)$ value is then estimated to be about $32 \mathrm{~T}$. Here, we note that the $H_{c 2}$ value would be even larger if the magnetic field is along the $c$ axis of a crystal. On the other hand, the Pauli paramagnetic limit for the upper critical field is $\mu_{0} H_{P}=1.84 T_{c} \approx 11 \mathrm{~T}$ in the case of an isotropic full superconducting gap without spin-orbit coupling [25,26]. Therefore, the $H_{c 2}(0)$ value could be over $300 \%-400 \%$ of the Pauli limit, which implies a novel spin-triplet Cooper pairing like that in the $p$-wave $\mathrm{SC}$ in $\mathrm{Sr}_{2} \mathrm{RuO}_{4}$ [27]. Further investigations using other techniques, such as nuclear magnetic resonance, are expected to be helpful to clarify this interesting issue.

The temperature dependence of specific heat $C(T)$ may supply important information for a superconductor. Figure 4 shows low- $T$ specific-heat data for the $\mathrm{K}_{2} \mathrm{Cr}_{3} \mathrm{As}_{3}$ polycrystals. The $C / T$ versus $T^{2}$ plot allows us to extract the contributions from electrons $\left(C_{e}=\gamma T\right)$ and phonons $\left(C_{\mathrm{ph}}=\beta T^{3}\right)$. The linear fit for the data from 6.4 to $10 \mathrm{~K}$ yields an electronic specific-heat coefficient $\gamma=70.2$ (for sample A) or 75.0 (for sample B) $\mathrm{mJ} \mathrm{K}^{-2} \mathrm{~mol} \mathrm{fu}^{-1}$ (fu refers to formula unit), equivalent to 23.4 or $25.0 \mathrm{~mJ} \mathrm{~K}^{-2} \mathrm{molCr}^{-1}$. This $\gamma$ value exceeds twice that $\left(9.1 \mathrm{~mJ} \mathrm{~K}^{-2} \mathrm{~mol} \mathrm{Cr}^{-1}\right)$ of the related three-dimensional correlated metal CrAs [28], indicating enhanced electron correlations in quasi-1D $\mathrm{K}_{2} \mathrm{Cr}_{3} \mathrm{As}_{3}$. The experimental density of states at the Fermi level, $N\left(E_{F}\right)=3 \gamma /\left(\pi k_{B}\right)^{2}=$ $30-32 \mathrm{eV}^{-1} \mathrm{fu}^{-1}\left(k_{B}\right.$ is the Boltzmann constant), is over 3 times that of the bare density of states $\left(8.58 \mathrm{eV}^{-1} \mathrm{fu}^{-1}\right)$ from the first-principles calculations [29]. This large renormalization factor cannot be explained by electronphonon interactions, and thus quantum fluctuations might be involved. With the fitted $\beta$ value of 1.57 (sample A) or 1.51 (sample B) $\mathrm{mJ} \mathrm{K}^{-4} \mathrm{~mol}^{-1}$, and using the formula

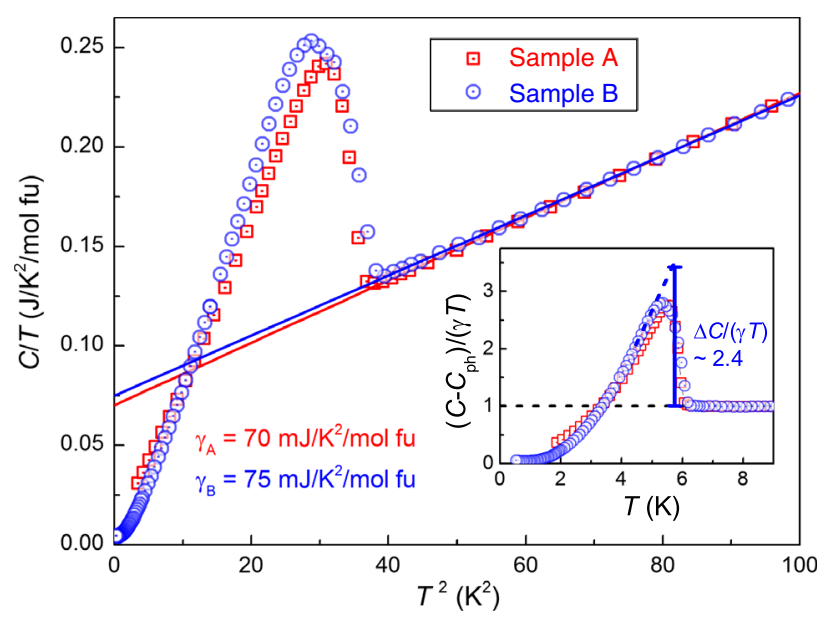

FIG. 4. Low-temperature specific heat for the $\mathrm{K}_{2} \mathrm{Cr}_{3} \mathrm{As}_{3}$ polycrystals. The main figure plots $C / T$ versus $T^{2}$, which extracts the contributions from phonons $\left(C_{\mathrm{ph}}=\beta T^{3}\right)$ and electrons $\left(C_{e}=\gamma T\right)$, and yields electronic specific-heat coefficients of $\gamma=70.2$ and $75.0 \mathrm{~mJ} \mathrm{~K}^{-2} \mathrm{molfu}^{-1}$, respectively, for sample $\mathrm{A}$ and sample B. The inset plots $\left(C-\beta T^{3}\right) /(\gamma T)$ as a function of temperature. 
$\theta_{D}=\left[(12 / 5) N R \pi^{4} / \beta\right]^{1 / 3}$, the Debye temperature can be calculated to be 215 and $218 \mathrm{~K}$.

Below $6 \mathrm{~K}$, a characteristic specific-heat jump $(\Delta C)$ due to the superconducting transition shows up, further confirming the bulk SC. Actually, $\Delta C$ comes from the change in the electronic part, $C_{e} \approx C-\beta T^{3}$ and, for revealing the $C_{e}(T)$ behavior in the superconducting state, $\left(C-\beta T^{3}\right)$ is normalized by the normal-state specific heat $\gamma T$ (see the inset of Fig. 4). As is seen, the dimensionless specific-heat jump at $T_{c}\left[\Delta C /\left(\gamma T_{c}\right)\right]$ is as high as 2.4 for sample $\mathrm{B}$, significantly larger than the theoretical value (1.43) of the well-known BCS theory, suggesting a strong coupling scenario. By the entropy-conserving construction, the thermodynamic transition temperature is determined to be $5.75 \mathrm{~K}$, coincident with the zero-resistance temperature. In general, the $C_{e}(T)$ dependence, which probes the lowenergy quasiparticle excitations, supplies information on the superconducting pairing symmetry. However, we find that the $\left(C-\beta T^{3}\right) /(\gamma T)$ data show an upturn below $1 \mathrm{~K}$, which is probably due to a Schottky-like anomaly from the related nuclei and/or impurities. This extra contribution prevents us from fitting the data to give a reliable conclusion.

\section{CONCLUDING REMARKS}

We discover superconductivity at $6.1 \mathrm{~K}$ under ambient pressure in a new $\mathrm{Cr}$-based material $\mathrm{K}_{2} \mathrm{Cr}_{3} \mathrm{As}_{3}$. The crystal structure is characterized by the $\left[\left(\mathrm{Cr}_{3} \mathrm{As}_{3}\right)^{2-}\right]_{\infty}$ chains, well separated by $\mathrm{K}^{+}$cations, which makes the title compound an explicitly quasi-1D material. Although the $T_{c}$ is not impressively high, to the best of our knowledge, however, it is among the highest in quasi-1D systems. The material also possesses a large electronic specific-heat coefficient of $70-75 \mathrm{~mJ} \mathrm{~K}^{-2} \mathrm{~mol}^{-1}$, indicating significantly strong electron correlations.

As a rare example of $\mathrm{SC}$ in a quasi-1D system with significant electron correlations, $\mathrm{K}_{2} \mathrm{Cr}_{3} \mathrm{As}_{3}$ also shows peculiar physical properties. The $T$-linear resistivity in a broad temperature range suggests a non-Fermi-liquid normal state, which could be related to a quantum criticality and/or realization of Luttinger liquid. The estimated $\mu_{0} H_{c 2}(0)$ value, even for the polycrystalline sample, is 3-4 times the Pauli limit [in comparison, the $\mu_{0} H_{c 2}(0)$ values of the quasi-1D $4.2 \mathrm{~K}$ superconductor $\mathrm{TlMo}_{3} \mathrm{Se}_{3}$ are 5.8 and $0.47 \mathrm{~T}$, respectively, for fields parallel and perpendicular to the $c$ axis [30]]. This is obviously beyond the explanation of the spin-orbit scattering and multiband effect, and thus a spin-triplet pairing is likely.

The appearance of $\mathrm{SC}$ in quasi-1D $\mathrm{K}_{2} \mathrm{Cr}_{3} \mathrm{As}_{3}$ is an unexpected result. First, the tendency of a Peierls transition in quasi-1D metals always prevents it from being superconductive. For example, most members of the aforementioned quasi-1D compounds $\mathrm{AkMo}_{3} \mathrm{Ch}_{3}[15,16]$, including $\mathrm{Ak}=\mathrm{K}$, do not show $\mathrm{SC}$ (exceptions are for $\mathrm{Ak}=\mathrm{Tl}$ and
In). Second, chromium is the only element metal that shows antiferromagnetism above room temperature, and it is one of the minor metallic elements that does not superconduct even under high pressures. While a few alloys containing Cr superconduct [31], SC in Cr-based compounds was not reported until the very recent observation of $\mathrm{SC}$ at $\sim 2 \mathrm{~K}$ in a three-dimensional compound CrAs under high pressures $[32,33]$. Therefore, $\mathrm{K}_{2} \mathrm{Cr}_{3} \mathrm{As}_{3}$ is the first Cr-based superconductor at ambient pressure. The occurrence of SC in this quasi-1D Cr-based material calls for further investigations.

\section{ACKNOWLEDGMENTS}

We thank H. Chen for his constructive suggestions on the single-crystal diffraction experiment. Thanks are also due to F. C. Zhang, C. Cao, Y. Zhou, J. H. Dai, and H. Q. Yuan for helpful discussions. This work was supported by the Natural Science Foundation of China (No. 11190023), the National Basic Research Program of China (No. 2011CBA00103), and the Fundamental Research Funds for the Central Universities of China.

Note added.-Most of the results presented in this work have been later confirmed by Kong, Bud'ko, and Canfield [34]. However, instead of the $T$-linear resistivity we observed in the polycrystalline samples, Ref. [34] reported a $T^{3}$ dependence from 10 to $40 \mathrm{~K}$ for single crystals. This discrepancy could be either due to sample deterioration of the single crystals or from a "polycrystal effect" [18]. We note that the $T$-linear resistivity in polycrystals is also seen in the two sister compounds of $\mathrm{K}_{2} \mathrm{Cr}_{3} \mathrm{As}_{3}$, $\mathrm{Rb}_{2} \mathrm{Cr}_{3} \mathrm{As}_{3}$ [35] and $\mathrm{Cs}_{2} \mathrm{Cr}_{3} \mathrm{As}_{3}$ [36].

[1] J. G. Bednorz and K. A. Muller, Possible High- $T_{c}$ Superconductivity in the Ba-La-Cu-O System, Z. Phys. B 64, 189 (1986).

[2] Y. Maeno, H. Hashimoto, K. Yoshida, S. Nishizaki, T. Fujita, J. G. Bednorz, and F. Lichtenberg, Superconductivity in a Layered Perovskite without Copper, Nature (London) 372, 532 (1994).

[3] Y. Kamihara, T. Watanabe, M. Hirano, and H. Hosono, Iron-Based Layered Superconductor $\mathrm{LaO}_{1-x} \mathrm{~F}_{x} \mathrm{FeAs}$ $(x=0.05-0.12)$ with $T_{c}=26 \mathrm{~K}$, J. Am. Chem. Soc. 130, 3296 (2008).

[4] R.E. Peierls, Quantum Theory of Solids (Clarendon, Oxford, 1955).

[5] S. van Smaalen, The Peierls Transition in Low-Dimensional Electronic Crystals, Acta Crystallogr. Sect. A 61, 51 (2005).

[6] D. Jerome, A. Mazaud, M. Ribault, and K. Bechgaard, Superconductivity in a Synthetic Organic Conductor (TMTSF) ${ }_{2} \mathrm{PF}_{6}$, J. Phys. (Paris), Lett. 41, L95 (1980).

[7] M. Greenblatt, W. H. McCarroll, R. Neifeld, M. Croft, and J. V. Waszczak, Quasi Two-Dimensional ElectronicProperties of the Lithium Molybdenum Bronze, $\mathrm{Li}_{0.9} \mathrm{Mo}_{6} \mathrm{O}_{17}$, Solid State Commun. 51, 671 (1984). 
[8] E. Dagotto and T. M. Rice, Surprises on the Way from One- to Two-Dimensional Quantum Magnets: The Ladder Materials, Science 271, 618 (1996).

[9] M. Uehara, T. Nagata, J. Akimitsu, H. Takahashi, N. Mori, and K. Kinoshita, Superconductivity in the Ladder Material $\mathrm{Sr}_{0.4} \mathrm{Ca}_{13.6} \mathrm{Cu}_{24} \mathrm{O}_{41.84}$, J. Phys. Soc. Jpn. 65, 2764 (1996).

[10] T. Nagata, M. Uehara, J. Goto, J. Akimitsu, N. Motoyama, H. Eisaki, S. Uchida, H. Takahashi, T. Nakanishi, and N. Môri, Pressure-Induced Dimensional Crossover and Superconductivity in the Hole-Doped Two-Leg Ladder Compound $\mathrm{Sr}_{14-x} \mathrm{Ca}_{x} \mathrm{Cu}_{24} \mathrm{O}_{41}$, Phys. Rev. Lett. 81, 1090 (1998).

[11] J. M. Tranquada, B. J. Sternlieb, J. D. Axe, Y. Nakamura, and S. Uchida, Evidence for Stripe Correlations of Spins and Holes in Copper Oxide Superconductors, Nature (London) 375, 561 (1995).

[12] J.-H. Chu, J. G. Analytis, K. De Greve, P. L. McMahon, Z. Islam, Y. Yamamoto, and I. R. Fisher, In-Plane Resistivity Anisotropy in an Underdoped Iron Arsenide Superconductor, Science 329, 824 (2010).

[13] J. Voit, One-Dimensional Fermi Liquids, Rep. Prog. Phys. 58, 977 (1995).

[14] See Supplemental Material at http://link.aps.org/ supplemental/10.1103/PhysRevX.5.011013 for details on the determinations of the chemical composition and crystal structure of $\mathrm{K}_{2} \mathrm{Cr}_{3} \mathrm{As}_{3}$ and Tables S1-S5.

[15] M. Potel, R. Chevrel, M. Sergent, J. C. Armici, M. Decroux, and $\varnothing$. Fischer, New Pseudo-One-Dimensional Metals: $\mathrm{M}_{2} \mathrm{Mo}_{6} \mathrm{Se}_{6} \quad\left(M=\mathrm{Na}, \mathrm{In}, \mathrm{K}, \mathrm{Tl}, \mathrm{M}_{2} \mathrm{Mo}_{6} \mathrm{Se}_{6}\right.$ $\left(M=\mathrm{K}, \mathrm{Rb}, \mathrm{Cs}, \mathrm{M}_{2} \mathrm{Mo}_{6} \mathrm{Te}_{6}(M=\mathrm{In}, \mathrm{Tl}), \mathrm{J}\right.$. Solid State Chem. 35, 286 (1980).

[16] W. Hönle, H. G. Von Schnering, A. Lipka, and K. Yvon, New Compounds with Infinite Chains of Face-Condensed Octahedral $\mathrm{Mo}_{6}$ Clusters: $\mathrm{InMo}_{3} \mathrm{Se}_{3}, \mathrm{InMo}_{3} \mathrm{Te}_{3}, \mathrm{TlMo}_{3} \mathrm{Se}_{3}$ and $\mathrm{TlMo}_{3} \mathrm{Te}_{3}$, J. Less-Common Met. 71, 135 (1980).

[17] Our preliminary measurements on a single stick of crystal are unreproducible, accompanied with deterioration of superconductivity. It is probably because of the use of silver paste (for making electrodes), which could react with the whole tiny crystal. For the polycrystalline pellets, however, the measurements can be reproducible, primarily because only a surface layer is damaged by the silver paste.

[18] We find that exposure of the sample in air for a short time could lead to deviation of the linearity, accompanied by a reduction of $T_{c}$. This leads us to believe that the linear- $T$ resistivity is an intrinsic property of the $\mathrm{K}_{2} \mathrm{Cr}_{3} \mathrm{As}_{3}$ polycrystals, which could mainly reflect the $\rho_{\|}(T)$ (resistivity along the chain direction) behavior. Nevertheless, the linear $\rho(T)$ might also be due to an incidental "polycrystal effect"; i.e., the as-measured linear $\rho(T)$ is a combined result of $\rho_{\|}(T), \rho_{\perp}(T)$, and the grain boundaries. Thus, the intrinsic $\rho_{\|}(T)$ and $\rho_{\perp}(T)$ need to be examined by using the singlecrystal sample without any deterioration.

[19] J. C. Armici, M. Decroux, Ø. Fischer, M. Potel, R. Chevrel, and M. Sergent, A New Pseudo-One-Dimensional Superconductor- $\mathrm{Tl}_{2} \mathrm{Mo}_{6} \mathrm{Se}_{6}$, Solid State Commun. 33, 607 (1980).

[20] S. Jiang, H. Xing, G. F. Xuan, C. Wang, Z. Ren, C. M. Feng, J. H. Dai, Z. A. Xu, and G. H. Cao, Superconductivity up to $30 \mathrm{~K}$ in the Vicinity of the Quantum Critical Point in
$\mathrm{BaFe}_{2}\left(\mathrm{As}_{1-x} \mathrm{P}_{x}\right)_{2}$, J. Phys. Condens. Matter 21, 382203 (2009).

[21] G. Stewart, Non-Fermi-Liquid Behavior in d-and f-Electron Metals, Rev. Mod. Phys. 73, 797 (2001).

[22] P. Khalifah, K. D. Nelson, R. Jin, Z. Q. Mao, Y. Liu, Q. Huang, X. P. A. Gao, A. P. Ramirez, and R. J. Cava, NonFermi-Liquid Behaviour in $\mathrm{La}_{4} \mathrm{Ru}_{6} \mathrm{O}_{19}$, Nature (London) 411, 669 (2001).

[23] M. Ogata and P. W. Anderson, Transport Properties in the Tomonaga-Luttinger Liquid, Phys. Rev. Lett. 70, 3087 (1993).

[24] N. R. Werthame, E. Helfand, and P. C. Hohenber, Temperature and Purity Dependence of Superconducting Critical Field $H_{c 2}$. III. Electron Spin and Spin-Orbit Effects, Phys. Rev. 147, 295 (1966).

[25] A. M. Clogston, Upper Limit for Critical Field in Hard Superconductors, Phys. Rev. Lett. 9, 266 (1962).

[26] B.S. Chandrasekhar, A Note on the Maximum Critical Field of High-Field Superconductors, Appl. Phys. Lett. 1, 7 (1962).

[27] K. D. Nelson, Z. Q. Mao, Y. Maeno, and Y. Liu, OddParity Superconductivity in $\mathrm{Sr}_{2} \mathrm{RuO}_{4}$, Science 306, 1151 (2004).

[28] W. Wu, X. Zhang, Z. Yin, P. Zheng, N. Wang, and J. Luo, Low Temperature Properties of Pnictide CrAs Single Crystal, Sci. China Phys. Mech. Astron. 53, 1207 (2010).

[29] H. Jiang, G. H. Cao, and C. Cao, Electronic Structure of Quasi-One-Dimensional Superconductor $\mathrm{K}_{2} \mathrm{Cr}_{3} \mathrm{As}_{3}$ from First-Principles Calculations, arXiv:1412.1309.

[30] A. P. Petrović, R. Lortz, G. Santi, M. Decroux, H. Monnard, O. Fischer, L. Boeri, O. K. Andersen, J. Kortus, D. Salloum, P. Gougeon, and M. Potel, Phonon Mode Spectroscopy, Electron-Phonon Coupling, and the Metal-Insulator Transition in Quasi-One-Dimensional $M_{2} \mathrm{Mo}_{6} \mathrm{Se}_{6}$, Phys. Rev. B 82, 235128 (2010).

[31] B. Matthias, T. Geballe, V. Compton, E. Corenzwit, and G. Hull, Superconductivity of Chromium Alloys, Phys. Rev. 128, 588 (1962).

[32] W. Wu, J. Cheng, K. Matsubayashi, P. Kong, F. Lin, C. Jin, N. Wang, Y. Uwatoko, and J. Luo, Superconductivity in the Vicinity of Antiferromagnetic Order in CrAs, Nat. Commun. 5, 5508 (2014).

[33] H. Kotegawa, S. Nakahara, H. Tou, and H. Sugawara, Superconductivity of $2.2 \mathrm{~K}$ under Pressure in Helimagnet CrAs, J. Phys. Soc. Jpn. 83, 093702 (2014).

[34] T. Kong, S. L. Bud'ko, and P. C. Canfield, Anisotropic $H_{c 2}$, Thermodynamic and Transport Measurements, and Pressure Dependence of $T_{c}$ in $\mathrm{K}_{2} \mathrm{Cr}_{3} \mathrm{As}_{3}$ Single Crystals, Phys. Rev. B 91, 020507(R) (2015).

[35] Z. T. Tang, J. K. Bao, Y. Liu, Y. L. Sun, H. F. Ablimit, A. Zhai, H. Jiang, C. M. Feng, Z. A. Xu, and G. H. Cao, Unconventional Superconductivity in Quasi-OneDimensional $\mathrm{Rb}_{2} \mathrm{Cr}_{3} \mathrm{As}_{3}$, Phys. Rev. B 91, 020506(R) (2015).

[36] Z. T. Tang, J. K. Bao, Z. Wang, H. Bai, H. Jiang, Y. Liu, H. F. Zhai, C. M. Feng, Z. A. Xu, and G. H. Cao, Superconductivity in Quasi-One-Dimensional $\mathrm{Cs}_{2} \mathrm{Cr}_{3} \mathrm{As}_{3}$ with Large Interchain Distance, Sci. China Mater. 58, 16 (2015). 